\title{
Adiponectin Provides Cardiovascular Protection in Metabolic Syndrome
}

\author{
Yoshihisa Okamoto \\ Department of Bioregulation, Nippon Medical School, 1-396 Kosugi-machi, Nakahara-ku, Kawasaki, Kanagawa 211-8533, Japan \\ Correspondence should be addressed to Yoshihisa Okamoto, yokamoto@nms.ac.jp \\ Received 24 November 2010; Accepted 19 December 2010 \\ Academic Editor: Rei Shibata \\ Copyright () 2011 Yoshihisa Okamoto. This is an open access article distributed under the Creative Commons Attribution License, \\ which permits unrestricted use, distribution, and reproduction in any medium, provided the original work is properly cited.
}

\begin{abstract}
Adipose tissue plays a central role in the pathogenesis of metabolic syndrome. Adiponectin (APN) is a bioactive adipocytokine secreted from adipocytes. Low plasma APN levels (hypoadiponectinemia) are observed among obese individuals and in those with related disorders such as diabetes, hypertension, and dyslipidemia. APN ameliorates such disorders. Hypoadiponectinemia is also associated with major cardiovascular diseases including atherosclerosis and cardiac hypertrophy. Accumulating evidence indicates that APN directly interacts with cardiovascular tissue and prevents cardiovascular pathology. Increasing plasma APN or enhancing APN signal transduction may be an ideal strategy to prevent and treat the cardiovascular diseases associated with metabolic syndrome. However, further studies are required to uncover the precise biological actions of APN.
\end{abstract}

\section{Introduction}

Obesity is one of the most common disorders in industrialized countries and is fast becoming a worldwide health problem. Metabolic disorders such as hypertension, dyslipidemia, and glucose intolerance frequently, but not incidentally, cluster in an individual with obesity, resulting in atherosclerotic cardiovascular diseases. This pathophysiology, based on excess visceral fat accumulation, has been conceptualized as "syndrome X," "deadly quartet," or "visceral fat syndrome," which are currently recognized as "metabolic syndrome [1]."

Adipose tissue plays a pivotal role in metabolic syndrome. Accumulating evidence indicates that adipose tissue secretes a variety of bioactive adipocytokines such as tumor necrosis factor $(\mathrm{TNF} \alpha)$, plasminogen activator inhibitor type 1 , retinol binding protein-4, monocyte chemotactic protein-1, and adiponectin (APN). Of these, APN has been cloned and is the most abundant. In the past decade, a large number of clinical and experimental studies have uncovered a variety of biological functions for APN. This paper updates the protective roles of APN in cardiovascular diseases and discusses the association of APN with metabolic syndrome.

\section{Clinical Features of Low Plasma APN (Hypoadiponectinemia)}

2.1. Obese Subjects and Patients with Coronary Risk Factors. The first clinical study of APN was conducted to observe plasma levels of APN among obese subjects. Although plasma levels of most other adipocytokines are higher in obese individuals, Arita et al. reported that plasma levels of APN are lower in obese individuals and are negatively correlated with body mass index (BMI) [2]. Subsequent studies demonstrated that plasma APN is lower (hypoadiponectinemia) in patients with diabetes, hypertension, and dyslipidemia than BMI-matched controls [3-5], indicating that hypoadiponectinemia is associated with an increased prevalence of coronary risk factors.

2.2. Coronary Artery Diseases. Subsequently, a series of clinical studies reported an association between hypoadiponectinemia and coronary artery diseases (CAD). Plasma APN is significantly lower in patients with CAD than control subjects, and male patients with hypoadiponectinemia have a twofold increase in CAD prevalence, independent of well- 
known risk factors [6, 7]. Another prospective study revealed that high plasma APN concentrations are associated with a lower risk for myocardial infarction in men, independent of inflammation and glycemic status [8]. Moreover, Otsuka et al. reported that patients with acute coronary syndrome have lower APN levels than patients with stable CAD and that plasma APN levels are significantly associated with coronary lesion complexity in men with CAD [9]. Several studies of patients undergoing percutaneous coronary intervention (PCI) indicate that hypoadiponectinemia is an independent predictor for in-stent restenosis [10, 11]. Recently, a multiple regression analysis revealed that levels of high molecular weight (HMW) APN correlate negatively with glycated hemoglobin in nondiabetic patients but positively with high-density lipoprotein cholesterol in diabetic patients with CAD [12]. These results indicate that total or HMW hypoadiponectinemia is an independent risk factor for CAD and that APN may directly protect against abnormal vascular remodeling.

2.3. Cardiac Diseases. Obesity is strongly associated with pathological cardiac remodeling, and several studies have investigated the association between plasma APN levels and cardiac diseases. Hypoadiponectinemia is associated with the progression of left ventricular hypertrophy (LVH) with diastolic dysfunction among patients with essential hypertension [13]. Even among healthy subjects, APN concentration is inversely and independently associated with LVH diagnosed by electrocardiography in Japanese men [14]. Another study using echocardiography revealed that circulating total APN and HMW APN are related to left ventricular wall thickness and diastolic function independent of age and metabolic factors [15]. These data suggest that APN may regulate hypertrophic progression of cardiomyocytes.

However, the role of APN in heart failure is controversial. Several studies have shown that plasma APN levels are high in patients with chronic heart failure (CHF) and are associated with CHF severity or mortality despite the protective effect of APN on CHF in mice [16-19]. $\beta$-blocker therapy correlates with lower APN levels in patients with $\mathrm{CHF}$, especially in nonobese patients, suggesting that this relationship should be considered when assessing plasma APN among patients with CHF [20]. Further careful studies may be required to clarify the relationship between APN and heart failure.

2.4. Chronic Kidney Disease (CKD). Increased albuminuria among patients with obesity and diabetes is a risk factor for cardiovascular and renal disease, and patients with CKD are at high risk for cardiovascular events. CKD patients show higher plasma APN levels than healthy subjects due to the low renal clearance rate of APN [21]. A prospective study of patients with renal failure demonstrated that patients who experience new cardiovascular events had lower plasma APN levels than event-free patients [22]. Several other studies have also indicated that increases in plasma APN in patients with CKD decrease their risk for cardiovascular disease and increase survival rate $[23,24]$. In contrast, a high APN level is associated with mortality, independent of risk markers for CHF severity among patients with CKD $[16,25]$. Therefore, the cardioprotective role of APN in CKD remains controversial. A recent report by Komura et al. revealed that the loss of the vascular protective function of APN in the presence of high cystatin C levels in patients with $\mathrm{CKD}$ indicates that cystatin $\mathrm{C}$ may mask the beneficial effect of APN in patients with CKD despite high plasma APN levels [21].

\section{Biological Features of APN}

\subsection{Atherosclerosis}

3.1.1. In Vitro. After initial clinical findings of the association between $\mathrm{CAD}$ and hypoadiponectinemia were reported, several experimental studies have been conducted to elucidate the biological effect of APN in atherosclerosis. APN has structural similarity with complimentary $\mathrm{Clq}$ or the collagen families [1]. APN specifically binds to collagen types I, III, and V, which are present in vascular intima and detected in the subendothelial space of rat balloon-injured arteries, implying an interaction between APN and vascular pathology [26].

When atherosclerosis commences, low-density lipoprotein (LDL) particles in the blood become oxidized (oxLDL) and induce the expression of adhesion molecules such as vascular cell adhesion molecule-1 (VCAM-1) and intercellular adhesion molecule-1, in endothelial cells (ECs). Leukocytes in blood attach to the endothelial layer and induce proinflammatory chemokines that attract leukocytes into the subendothelial space [27]. APN inhibits the expression of these adhesion molecules in TNF $\alpha$-activated endothelial cells by suppressing inflammatory transcriptional factors and activating nuclear factor (NF)- $\kappa \mathrm{B}[6,28]$. Additionally, APN inhibits TNF $\alpha$-inducible interleukin (IL)-8 synthesis in ECs by inactivating NF- $\kappa \mathrm{B}$ and activating Akt [29]. An in vivo study in mice demonstrated that APN deficiency increases leukocyte-endothelium interactions with impaired endothelial nitric oxide signaling (eNOS) via upregulation of endothelial cell adhesion molecules [30].

The recruited inflammatory cells subsequently enhance the oxidization of LDL and various inflammatory reactions. Monocytes migrate to the subendothelial space in the atherosclerotic lesion and become lipid-laden macrophages. Monocytes change into macrophages in the subendothelial space by taking up oxLDL via scavenger receptors and form cells with accumulated cholesterol esters. APN suppresses scavenger receptor type A (SR-A) in macrophages and the internal cholesterol ester content. However, macrophages play an important role in reverse cholesterol transport (RCT), a protective system against atherosclerosis. APN increases apoA-I-mediated cholesterol efflux from macrophages through an ATP-binding cassette transporter A1-dependent pathway, indicating that APN may prevent atherosclerosis by accelerating RCT [31]. Macrophages sustain and amplify the inflammatory process by releasing several growth factors, cytokines, and chemokines that may further recruit immune cells, including monocyte/ 
macrophages, T lymphocytes, or vascular smooth muscle cells. Pretreatment with recombinant APN significantly suppresses the production of cytokines/chemokines, such as TNF $\alpha$, and CXCR3 ligand chemokines, such as IFNinducible protein of $10 \mathrm{kDa}$ (IP-10), monokine induced by IFN- $\gamma$, and IFN-inducible T cells, which is a chemoattractant in lipopolysaccharide-stimulated macrophages [32, 33]. The inflammatory process includes enzymes that can destroy the arterial extracellular matrix such as metalloproteinases (MMPs). APN treatment also induces anti-inflammatory IL10 and subsequent tissue inhibition of MMP-1 production, suggesting that APN may stabilize atherosclerotic plaques and prevent their rupture [34].

Aortic smooth muscle cells (AoSMCs) are another major player in atherosclerosis, and their pathological migration and proliferation in the intima relates to restenosis of coronary arteries after PCI. APN suppresses growth factorstimulated AoSMC proliferation and migration by inhibiting the ERK signal [35].

3.1.2. In Vivo. Several experimental studies have demonstrated the effect of APN on atherogenesis. The increment of total APN or globular APN significantly attenuates the progression of atherosclerosis in apoE knockout mice [36, 37]. In atherosclerotic lesions, APN accumulates to form cells in fatty streaks and inhibits the expression of VCAM1, SR-A, and TNF $\alpha$ [36]. In addition, APN/apoE double knockout mice show advanced atherosclerotic lesions with increased T-lymphocyte accumulation and higher plasma IP-10 levels compared with apoE single knockout mice (Figure 1) [33]. Moreover, APN deficiency worsens neointimal formation after endothelial injury in mice, while APN supplements reverse the abnormal vascular remodeling [38]. These data support the in vitro bioactivity of APN as an antiinflammatory adipocytokine in the atherosclerotic process.

3.2. Cardiac Diseases. Clinical studies have demonstrated that APN is associated with myocardial pathophysiology. APN deficiency causes severe concentric cardiac hypertrophy in mice after pressure overload with increased extracellular signal-regulated kinase, diminishes AMP-activated protein kinase (AMPK) signaling in the myocardium, and increases mortality [39]. Supplementing APN with an adenovirus vector attenuates the pathological cardiac hypertrophy. In an ischemia/reperfusion myocardium injury model, APN knockout mice exhibit increases in myocardial infarct size, apoptosis, and TNF $\alpha$ expression compared with controls. In cultured cardiomyocytes, APN inhibits $\mathrm{TNF} \alpha$ production by inducing cyclooxygenase-2-dependent synthesis of prostaglandin E 2 [40]. A similar experiment showed that globular APN protects myocardium from ischemia/reperfusion injury by inhibiting inducible NOS and nicotinamide adenine dinucleotide phosphate oxidase protein expression and resultant oxidative/nitrate stress [41]. Two recent reports demonstrated that APN knockout mice show enhanced cardiac fibrosis following permanent ligation of the left anterior descending artery or angiotensin II infusion. APN accumulates in the injured cardiomyocytes and protects it against fibrosis by reducing apoptosis and AMPK-dependent peroxisome proliferator-activated receptor $(\operatorname{PPAR} \alpha)$ activation $[19,42]$. Despite the potential association between heart failure and high plasma APN shown by several clinical studies, other experimental studies have demonstrated a beneficial, protective effect of APN on myocardium. Elevated plasma levels of APN among patients with heart failure can be a reflection of accompanying renal dysfunction or "APN resistance" including impaired APN signal transduction in myocardium.

3.3. APN and Pulmonary Artery Remodeling. Pulmonary arterial hypertension (PAH) is an idiopathic disease characterized by an increase in the thickness of pulmonary artery wall. APN suppresses platelet-derived growth factor BBmediated proliferation of pulmonary artery smooth muscle cells harvested from mice [43], indicating that APN may play a role in the prevention of PAH. In a mouse model of chronic airway inflammation, APN deficiency causes pathological pulmonary arterial wall thickness and elevates right ventricular systolic pressure, indicating PAH [44]. APN knockout mice also show thickening of pulmonary arterial wall under chronic hypoxic exposure, and APN overexpression significantly decreases the wall remodeling and right ventricular hypertrophy [45].

\subsection{APN and Endothelial Function, Angiogenesis, and Hyper-} tension. APN serves as an angiogenic factor. In a mouse hindlimb ischemia model, APN deficiency impairs revascularization, whereas adenovirus-mediated APN administration recovers angiogenesis [46]. APN stimulates blood vessel growth in the in vivo mouse Matrigel plug implantation and rabbit corneal models of angiogenesis by promoting crosstalk between AMP-activated protein kinase and Akt signaling within endothelial cells [47]. APN also dose dependently suppresses endothelial cell apoptosis and proliferation, migration, and premature diabetic senescence of endothelial progenitor cells [48-50].

Hypoadiponectinemia is associated with impaired endothelial dysfunction related to vasorelaxation. APN knockout mice show a significantly reduced endothelium-dependent vasodilation in response to acetylcholine compared with wild-type mice [51]. Another experiment demonstrated that APN knockout mice develop hypertension when maintained on a high-salt diet $(8 \% \mathrm{NaCl})$ without insulin resistance [52]. Notably, all of these APNs protective effects on endothelial function are mediated through an increase in the production of eNOS [51, 53-55].

\subsection{APN Strategies for Cardiovascular Protection in Metabolic Syndrome. Hypoadiponectinemia directly promotes the pathological reactions in cardiovascular system (Figure 2). Therefore, the increment of plasma APN is important to maximize the beneficial effects of APN. A reduction in body weight, especially visceral fat mass, with a combination of diet therapy and exercise is a safe and effective way to increase plasma APN levels.}




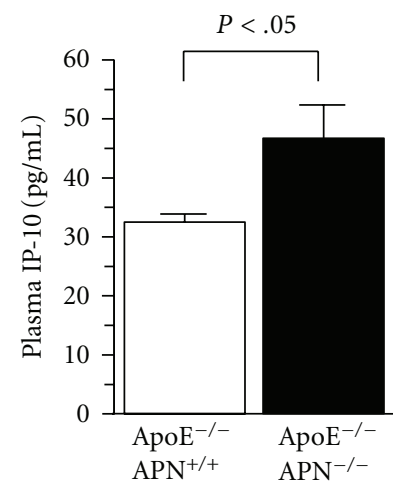

(a)

Oil Red O
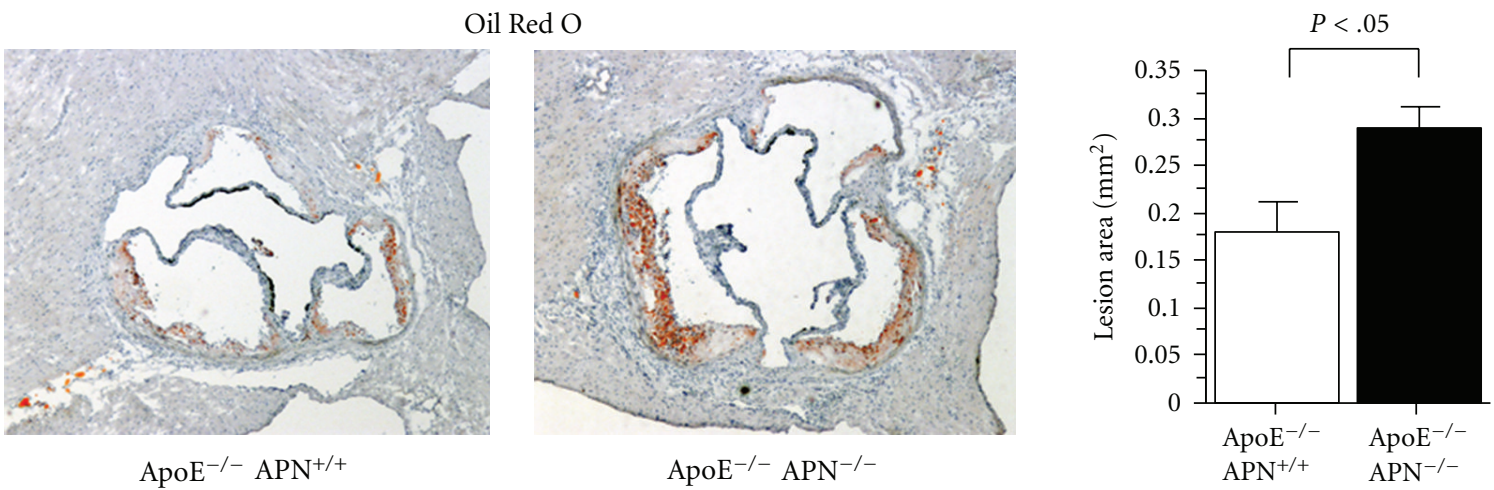

(b)
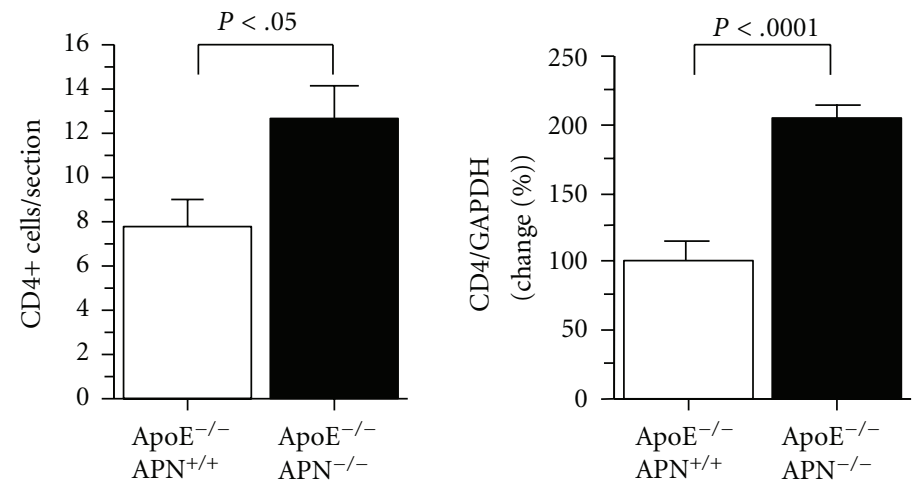

(c)

Figure 1: APN deficiency in apoE knockout mice. Compared with apoE single knockout mice, APN/apoE double knockout mice showed (a) higher plasma IP-10 levels, (b) advanced atherosclerosis, and (c) accelerated accumulation of T lymphocytes in atherosclerotic lesions (Adapted from [33]).

Another strategy to prevent cardiovascular disease using APN includes pharmacological changes in plasma APN levels. PPAR $\gamma$ agonists significantly increase plasma APN concentrations in insulin-resistant humans without affecting their body weight and in a mouse model of oxygen-induced retinopathy $[56,57]$. Administering PPAR $\alpha$ ligands and angiotensin receptor blockers also increases plasma APN levels $[53,58-60]$. Furthermore, several statins are also effective for elevating plasma APN [61, 62]. Changes in HMW APN as well as total APN may also be an ideal target. The natriuretic peptides including atrial natriuretic peptide (ANP) and brain natriuretic peptide (BNP) also increased the production of APN in adipocytes and plasma APN levels among patients with congestive heart failure [63]. Exogenous administration of adiponectin may be a therapeutic strategy as well. In a pig model, a single intracoronary administration of recombinant adiponectin protected myocardial ischemia/reperfusion injury by suppressing inflammation, apoptosis and oxidative stress [64].

APN receptors or candidate APN-receptor-binding proteins have been reported such as AdipoR1-R2, T-cadherin, and calreticulin [65-67]. Very recently, Denzel et al. reported that T-cadherin (glycosyl phosphatidylinositol-anchored cell surface glycoprotein) is critical for adiponectin-mediated cardioprotection by showing no effect of adenovirusmediated adiponectin supplement in T-cadherin knockout 


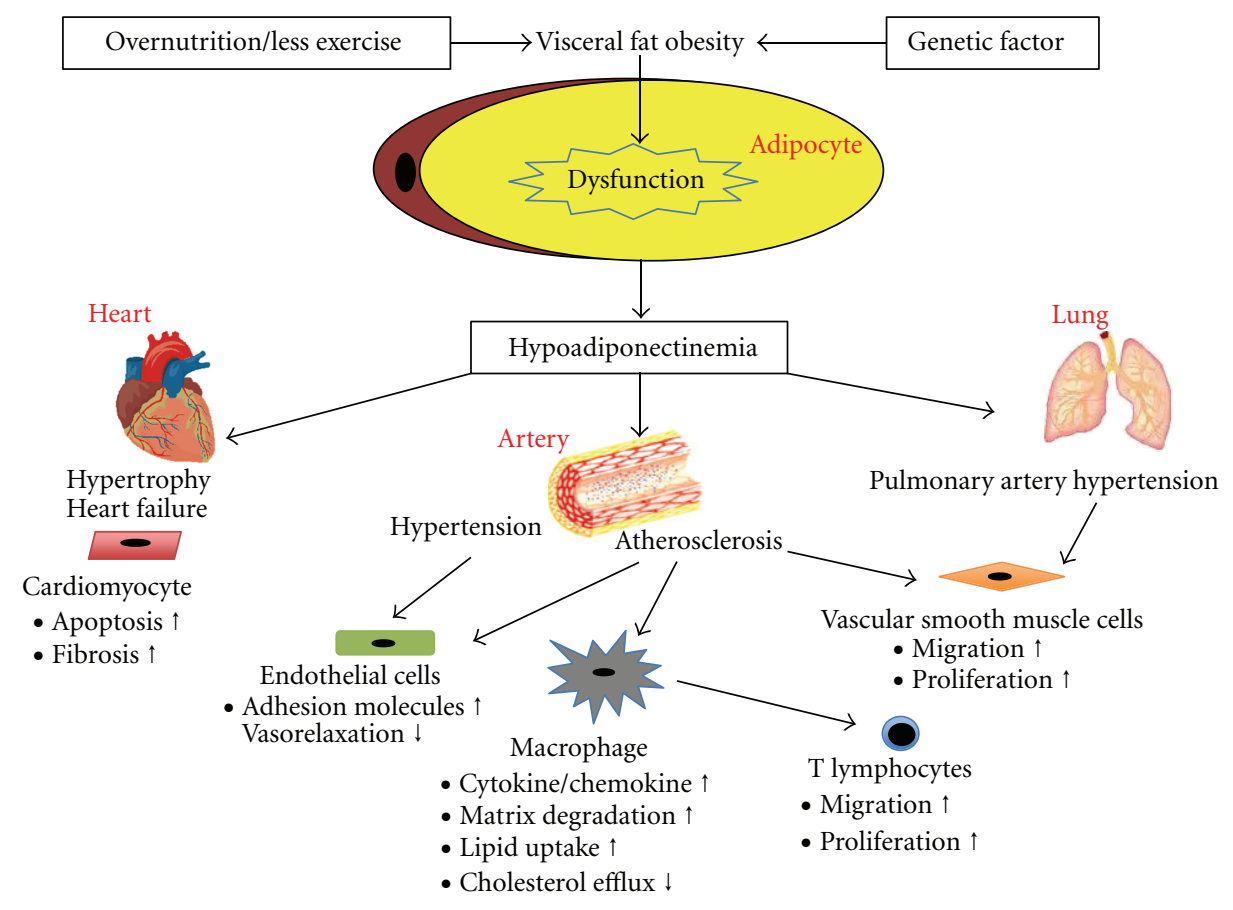

FIGURE 2: Hypoadiponectinemia directly promotes the pathological reactions in cardiovascular system.

mice [68]. Pharmacologically enhancing the expression of or activating APN receptors may be a good strategy for cardiovascular protection.

\section{Conclusion}

Adipose tissue stores not only excess body energy but also a variety of adipocytokines that regulate cardiovascular homeostasis directly and indirectly. Accumulating evidence has demonstrated that APN prevents diabetes, dyslipidemia, and hypertension, which are well-known risk factors for cardiovascular disease. Notably, APN directly interacts with cardiac and vascular tissues and mitigates pathological reactions. Generally, anti-inflammation is a key biological action of APN for cardiovascular protection. Further clinical and experimental studies will clarify the precise effects and mechanisms of APN action for future clinical use.

\section{Acknowledgment}

This work was supported by a grant from the Takeda Science Foundation.

\section{References}

[1] Y. Okamoto, S. Kihara, T. Funahashi, Y. Matsuzawa, and P. Libby, "Adiponectin: a key adipocytokine in metabolic syndrome," Clinical Science, vol. 110, no. 3, pp. 267-278, 2006.

[2] Y. Arita, S. Kihara, N. Ouchi et al., "Paradoxical decrease of an adipose-specific protein, adiponectin, in obesity," Biochemical and Biophysical Research Communications, vol. 257, no. 1, pp. 79-83, 1999.
[3] K. Hotta, T. Funahashi, N. L. Bodkin et al., "Circulating concentrations of the adipocyte protein adiponectin are decreased in parallel with reduced insulin sensitivity during the progression to type 2 diabetes in rhesus monkeys," Diabetes, vol. 50, no. 5, pp. 1126-1133, 2001.

[4] Y. Iwashima, T. Katsuya, K. Ishikawa et al., "Hypoadiponectinemia is an independent risk factor for hypertension," Hypertension, vol. 43, no. 6, pp. 1318-1323, 2004.

[5] M. Matsubara, S. Maruoka, and S. Katayose, "Decreased plasma adiponectin concentrations in women with dyslipidemia," Journal of Clinical Endocrinology and Metabolism, vol. 87, no. 6, pp. 2764-2769, 2002.

[6] N. Ouchi, S. Kihara, Y. Arita et al., "Novel modulator for endothelial adhesion molecules: adipocyte-derived plasma protein adiponectin," Circulation, vol. 100, no. 25, pp. 24732476, 1999.

[7] M. Kumada, S. Kihara, S. Sumitsuji et al., "Association of hypoadiponectinemia with coronary artery disease in men," Arteriosclerosis, Thrombosis, and Vascular Biology, vol. 23, no. 1, pp. 85-89, 2003.

[8] T. Pischon, C. J. Girman, G. S. Hotamisligil, N. Rifai, F. B. $\mathrm{Hu}$, and E. B. Rimm, "Plasma adiponectin levels and risk of myocardial infarction in men," Journal of the American Medical Association, vol. 291, no. 14, pp. 1730-1737, 2004.

[9] F. Otsuka, S. Sugiyama, S. Kojima et al., "Plasma adiponectin levels are associated with coronary lesion complexity in men with coronary artery disease," Journal of the American College of Cardiology, vol. 48, no. 6, pp. 1155-1162, 2006.

[10] Y. Kitta, H. Takano, T. Nakamura et al., "Low adiponectin levels predict late in-stent restenosis after bare metal stenting in native coronary arteries," International Journal of Cardiology, vol. 131, no. 1, pp. 78-82, 2008.

[11] E. Moldoveanu, B. Mut-Vitcu, G. R. Tanaseanu et al., "Low basal levels of circulating adiponectin in patients undergoing coronary stenting predict in-stent restenosis, independently of 
basal levels of inflammatory markers: lipoprotein associated phospholipase A2, and myeloperoxidase," Clinical Biochemistry, vol. 41, no. 18, pp. 1429-1433, 2008.

[12] N. Komura, S. Kihara, M. Sonoda et al., "Clinical significance of high-molecular weight form of adiponectin in male patients with coronary artery disease," Circulation Journal, vol. 72, no. 1, pp. 23-28, 2008.

[13] S. J. Hong, C. G. Park, H. S. Seo, D. J. Oh, and Y. M. Ro, "Associations among plasma adiponectin, hypertension, left ventricular diastolic function and left ventricular mass index," Blood Pressure, vol. 13, no. 4, pp. 236-242, 2004.

[14] H. Mitsuhashi, H. Yatsuya, K. Tamakoshi et al., "Adiponectin level and left ventricular hypertrophy in Japanese men," Hypertension, vol. 49, no. 6, pp. 1448-1454, 2007.

[15] M. Kozakova, E. Muscelli, A. Flyvbjerg et al., "Adiponectin and left ventricular structure and function in healthy adults," Journal of Clinical Endocrinology and Metabolism, vol. 93, no. 7, pp. 2811-2818, 2008.

[16] C. Kistorp, J. Faber, S. Galatius et al., "Plasma adiponectin, body mass index, and mortality in patients with chronic heart failure," Circulation, vol. 112, no. 12, pp. 1756-1762, 2005.

[17] T. Nakamura, H. Funayama, N. Kubo et al., "Association of hyperadiponectinemia with severity of ventricular dysfunction in congestive heart failure," Circulation Journal, vol. 70, no. 12, pp. 1557-1562, 2006.

[18] T. Tamura, Y. Furukawa, R. Taniguchi et al., "Serum adiponectin level as an independent predictor of mortality in patients with congestive heart failure," Circulation Journal, vol. 71, no. 5, pp. 623-630, 2007.

[19] R. Shibata, Y. Izumiya, K. Sato et al., "Adiponectin protects against the development of systolic dysfunction following myocardial infarction," Journal of Molecular and Cellular Cardiology, vol. 42, no. 6, pp. 1065-1074, 2007.

[20] A. Biolo, R. Shibata, N. Ouchi et al., "Determinants of adiponectin levels in patients with chronic systolic heart failure," American Journal of Cardiology, vol. 105, no. 8, pp. $1147-1152,2010$.

[21] N. Komura, S. Kihara, M. Sonoda et al., "Increment and impairment of adiponectin in renal failure," Cardiovascular Research, vol. 86, no. 3, pp. 471-477, 2010.

[22] C. Zoccali, F. Mallamaci, G. Tripepi et al., "Adiponectin, metabolic risk factors, and cardiovascular events among patients with end-stage renal disease," Journal of the American Society of Nephrology, vol. 13, no. 1, pp. 134-141, 2002.

[23] Y. Iwashima, T. Horio, M. Kumada et al., "Adiponectin and renal function, and implication as a risk of cardiovascular disease," American Journal of Cardiology, vol. 98, no. 12, pp. 1603-1608, 2006.

[24] J. J. Díez, P. Estrada, M. A. Bajo et al., "High stable serum adiponectin levels are associated with a better outcome in prevalent dialysis patients," American Journal of Nephrology, vol. 30, no. 3, pp. 244-252, 2009.

[25] V. Menon, L. Li, X. Wang et al., "Adiponectin and mortality in patients with chronic kidney disease," Journal of the American Society of Nephrology, vol. 17, no. 9, pp. 2599-2606, 2006.

[26] Y. Okamoto, Y. Arita, M. Nishida et al., "An adipocyte-derived plasma protein, adiponectin, adheres to injured vascular walls," Hormone and Metabolic Research, vol. 32, no. 2, pp. 4750, 2000.

[27] P. Libby, Y. Okamoto, V. Z. Rocha, and E. Folco, "Inflammation in atherosclerosis: transition from theory to practice," Circulation Journal, vol. 74, no. 2, pp. 213-220, 2010.

[28] N. Ouchi, S. Kihara, Y. Arita et al., "Adiponectin, an adipocytederived plasma protein, inhibits endothelial NF- $\kappa$ B signaling through a cAMP-dependent pathway," Circulation, vol. 102, no. 11, pp. 1296-1301, 2000.

[29] C. Kobashi, M. Urakaze, M. Kishida et al., "Adiponectin inhibits endothelial synthesis of interleukin-8," Circulation Research, vol. 97, no. 12, pp. 1245-1252, 2005.

[30] R. Ouedraogo, Y. Gong, B. Berzins et al., "Adiponectin deficiency increases leukocyte-endothelium interactions via upregulation of endothelial cell adhesion molecules in vivo," Journal of Clinical Investigation, vol. 117, no. 6, pp. 1718-1726, 2007.

[31] K. Tsubakio-Yamamoto, F. Matsuura, M. Koseki et al., "Adiponectin prevents atherosclerosis by increasing cholesterol efflux from macrophages," Biochemical and Biophysical Research Communications, vol. 375, no. 3, pp. 390-394, 2008.

[32] T. Yokota, K. Oritani, I. Takahashi et al., "Adiponectin, a new member of the family of soluble defense collagens, negatively regulates the growth of myelomonocytic progenitors and the functions of macrophages," Blood, vol. 96, no. 5, pp. 1723$1732,2000$.

[33] Y. Okamoto, E. J. Folco, M. Minami et al., "Adiponectin inhibits the production of CXC receptor 3 chemokine ligands in macrophages and reduces T-lymphocyte recruitment in atherogenesis," Circulation Research, vol. 102, no. 2, pp. 218225, 2008.

[34] M. Kumada, S. Kihara, N. Ouchi et al., "Adiponectin specifically increased tissue inhibitor of metalloproteinase-1 through interleukin-10 expression in human macrophages," Circulation, vol. 109, no. 17, pp. 2046-2049, 2004.

[35] Y. Arita, S. Kihara, N. Ouchi et al., "Adipocyte-derived plasma protein adiponectin acts as a platelet-derived growth factorBB-binding protein and regulates growth factor-induced common postreceptor signal in vascular smooth muscle cell," Circulation, vol. 105, no. 24, pp. 2893-2898, 2002.

[36] Y. Okamoto, S. Kihara, N. Ouchi et al., "Adiponectin reduces atherosclerosis in apolipoprotein E-deficient mice," Circulation, vol. 106, no. 22, pp. 2767-2770, 2002.

[37] T. Yamauchi, J. Kamon, H. Waki et al., "Globular adiponectin protected ob/ob mice from diabetes and ApoE-deficient mice from atherosclerosis," Journal of Biological Chemistry, vol. 278, no. 4, pp. 2461-2468, 2003.

[38] M. Matsuda, I. Shimomura, M. Sata et al., "Role of adiponectin in preventing vascular stenosis. The missing link of adipo-vascular axis," Journal of Biological Chemistry, vol. 277, no. 40, pp. 37487-37491, 2002.

[39] R. Shibata, N. Ouchi, M. Ito et al., "Adiponectin-mediated modulation of hypertrophic signals in the heart," Nature Medicine, vol. 10, no. 12, pp. 1384-1389, 2004.

[40] R. Shibata, K. Sato, D. R. Pimentel et al., "Adiponectin protects against myocardial ischemia-reperfusion injury through AMPK- and COX-2-dependent mechanisms," Nature Medicine, vol. 11, no. 10, pp. 1096-1103, 2005.

[41] L. Tao, E. Gao, X. Jiao et al., "Adiponectin cardioprotection after myocardial ischemia/reperfusion involves the reduction of oxidative/nitrative stress," Circulation, vol. 115, no. 11, pp. 1408-1416, 2007.

[42] K. Fujita, N. Maeda, M. Sonoda et al., "Adiponectin protects against angiotensin II-induced cardiac fibrosis through activation of PPAR- $\alpha$," Arteriosclerosis, Thrombosis, and Vascular Biology, vol. 28, no. 5, pp. 863-870, 2008.

[43] G. Hansmann, R. A. Wagner, S. Schellong et al., "Pulmonary arterial hypertension is linked to insulin resistance and reversed by peroxisome proliferator-activated receptor- $\gamma$ activation," Circulation, vol. 115, no. 10, pp. 1275-1284, 2007. 
[44] B. D. Medoff, Y. Okamoto, P. Leyton et al., "Adiponectin deficiency increases allergic airway inflammation and pulmonary vascular remodeling," American Journal of Respiratory Cell and Molecular Biology, vol. 41, no. 4, pp. 397-406, 2009.

[45] Y. Nakagawa, K. Kishida, S. Kihara, T. Funahashi, and I. Shimomura, "Adiponectin ameliorates hypoxia-induced pulmonary arterial remodeling," Biochemical and Biophysical Research Communications, vol. 382, no. 1, pp. 183-188, 2009.

[46] R. Shibata, N. Ouchi, S. Kihara, K. Sato, T. Funahashi, and $\mathrm{K}$. Walsh, "Adiponectin stimulates angiogenesis in response to tissue ischemia through stimulation of AMP-activated protein kinase signaling," Journal of Biological Chemistry, vol. 279, no. 27, pp. 28670-28674, 2004.

[47] N. Ouchi, H. Kobayashi, S. Kihara et al., "Adiponectin stimulates angiogenesis by promoting cross-talk between AMPactivated protein kinase and Akt signaling in endothelial cells," Journal of Biological Chemistry, vol. 279, no. 2, pp. 1304-1309, 2004.

[48] H. Kobayashi, N. Ouchi, S. Kihara et al., "Selective suppression of endothelial cell apoptosis by the high molecular weight form of adiponectin," Circulation research, vol. 94, no. 4, pp. e27-e31, 2004.

[49] R. Shibata, C. Skurk, N. Ouchi et al., "Adiponectin promotes endothelial progenitor cell number and function," FEBS Letters, vol. 582, no. 11, pp. 1607-1612, 2008.

[50] J. Chang, Y. Li, Y. Huang et al., "Adiponectin prevents diabetic premature senescence of endothelial progenitor cells and promotes endothelial repair by suppressing the p38 MAP kinase/p16INK4A signaling pathway," Diabetes, vol. 59, no. 11, pp. 2949-2959, 2010.

[51] N. Ouchi, M. Ohishi, S. Kihara et al., "Association of hypoadiponectinemia with impaired vasoreactivity," Hypertension, vol. 42, no. 3, pp. 231-234, 2003.

[52] K. Ohashi, S. Kihara, N. Ouchi et al., "Adiponectin replenishment ameliorates obesity-related hypertension," Hypertension, vol. 47, no. 6, pp. 1108-1116, 2006.

[53] A. Kurata, H. Nishizawa, S. Kihara et al., "Blockade of angiotensin II type-1 receptor reduces oxidative stress in adipose tissue and ameliorates adipocytokine dysregulation," Kidney International, vol. 70, no. 10, pp. 1717-1724, 2006.

[54] H. Chen, M. Montagnani, T. Funahashi, I. Shimomura, and M. J. Quon, "Adiponectin stimulates production of nitric oxide in vascular endothelial cells," Journal of Biological Chemistry, vol. 278, no. 45, pp. 45021-45026, 2003.

[55] K. K. Y. Cheng, K. S. L. Lam, Y. Wang et al., "Adiponectininduced endothelial nitric oxide synthase activation and nitric oxide production are mediated by APPL1 in endothelial cells," Diabetes, vol. 56, no. 5, pp. 1387-1394, 2007.

[56] N. Maeda, M. Takahashi, T. Funahashi et al., "PPAR $\gamma$ ligands increase expression and plasma concentrations of adiponectin, an adipose-derived protein," Diabetes, vol. 50, no. 9, pp. 20942099, 2001.

[57] A. Higuchi, K. Ohashi, R. Shibata, S. Sono-Romanelli, K. Walsh, and N. Ouchi, "Thiazolidinediones reduce pathological neovascularization in ischemic retina via an adiponectindependent mechanism," Arteriosclerosis, Thrombosis, and Vascular Biology, vol. 30, no. 1, pp. 46-53, 2010.

[58] A. Hiuge, A. Tenenbaum, N. Maeda et al., "Effects of peroxisome proliferator-activated receptor ligands, bezafibrate and fenofibrate, on adiponectin level," Arteriosclerosis, Thrombosis, and Vascular Biology, vol. 27, no. 3, pp. 635-641, 2007.

[59] N. Komiya, H. Hirose, H. Kawabe, H. Itoh, and I. Saito, "Effects of telmisartan therapy on metabolic profiles and serum high molecular weight (HMW)-adiponectin level in
Japanese male hypertensive subjects with abdominal obesity," Journal of Atherosclerosis and Thrombosis, vol. 16, no. 2, pp. 137-142, 2009.

[60] P. Li, R. Shibata, S. Maruyama et al., "Fenofibrate promotes ischemia-induced revascularization through the adiponectindependent pathway," American Journal of Physiology, vol. 299, no. 4, pp. E560-E566, 2010.

[61] T. Takagi, M. Matsuda, M. Abe et al., "Effect of pravastatin on the development of diabetes and adiponectin production," Atherosclerosis, vol. 196, no. 1, pp. 114-121, 2008.

[62] N. Inami, S. Nomura, A. Shouzu et al., "Effects of pitavastatin on adiponectin in patients with hyperlipidemia," Pathophysiology of Haemostasis and Thrombosis, vol. 36, no. 1, pp. 1-8, 2008.

[63] O. Tsukamoto, M. Fujita, M. Kato et al., "Natriuretic peptides enhance the production of adiponectin in human adipocytes and in patients with chronic heart failure," Journal of the American College of Cardiology, vol. 53, no. 22, pp. 2070-2077, 2009.

[64] K. Kondo, R. Shibata, K. Unno et al., "Impact of a single intracoronary administration of adiponectin on myocardial ischemia/reperfusion injury in a pig model," Circulation: Cardiovascular Interventions, vol. 3, no. 2, pp. 166-173, 2010.

[65] T. Yamauchi, Y. Nio, T. Maki et al., "Targeted disruption of AdipoR1 and AdipoR2 causes abrogation of adiponectin binding and metabolic actions," Nature Medicine, vol. 13, no. 3, pp. 332-339, 2007.

[66] C. Hug, J. Wang, N. S. Ahmad, J. S. Bogan, T. S. Tsao, and H. F. Lodish, "T-cadherin is a receptor for hexameric and highmolecular-weight forms of Acrp30/adiponectin," Proceedings of the National Academy of Sciences of the United States of America, vol. 101, no. 28, pp. 10308-10313, 2004.

[67] Y. Takemura, N. Ouchi, R. Shibata et al., "Adiponectin modulates inflammatory reactions via calreticulin receptordependent clearance of early apoptotic bodies," Journal of Clinical Investigation, vol. 117, no. 2, pp. 375-386, 2007.

[68] M. S. Denzel, M.-C. Scimia, P. M. Zumstein, K. Walsh, P. Ruiz-Lozano, and B. Ranscht, "T-cadherin is critical for adiponectin-mediated cardioprotection in mice," Journal of Clinical Investigation, vol. 120, no. 12, pp. 4342-4352, 2010. 


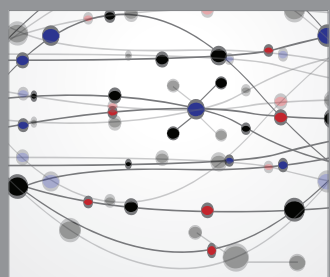

The Scientific World Journal
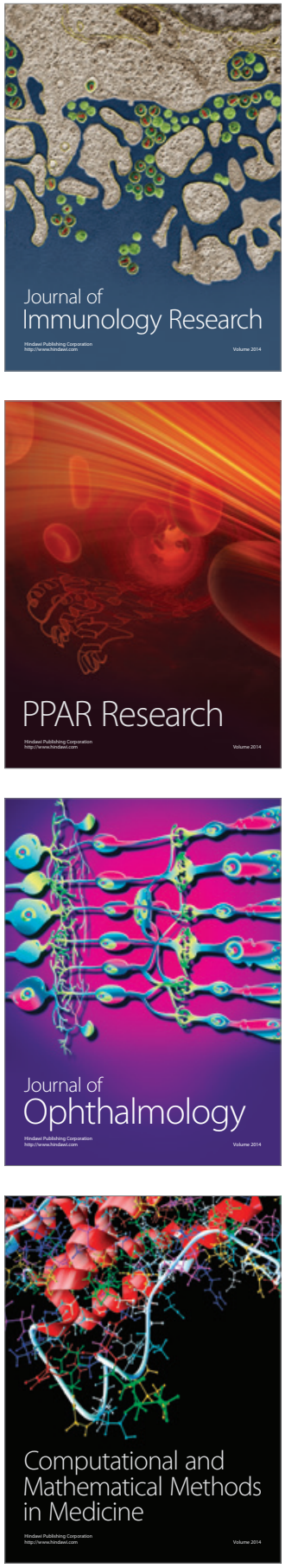

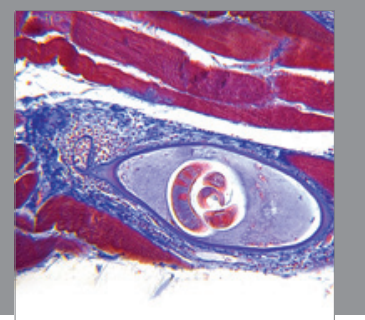

Gastroenterology

Research and Practice
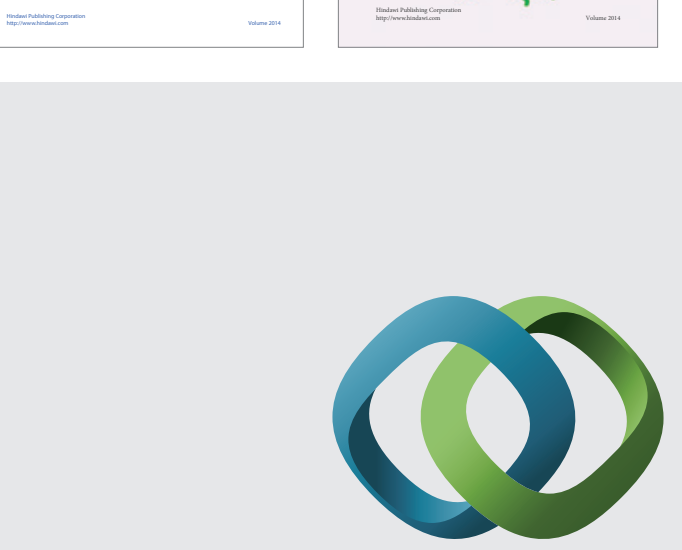

\section{Hindawi}

Submit your manuscripts at

http://www.hindawi.com
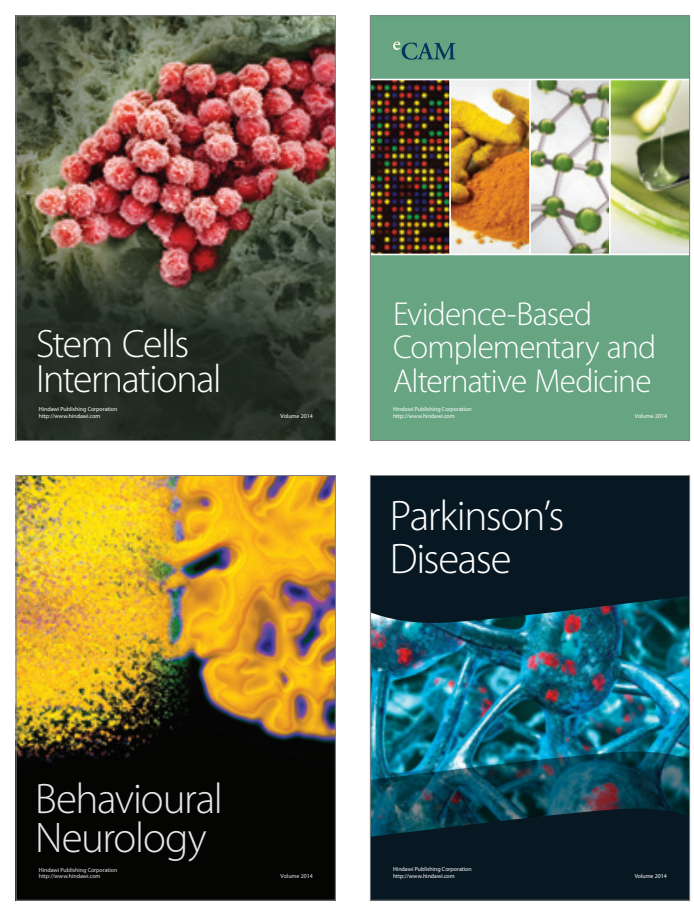

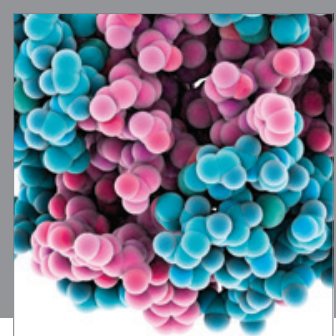

Journal of
Diabetes Research

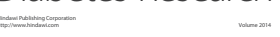

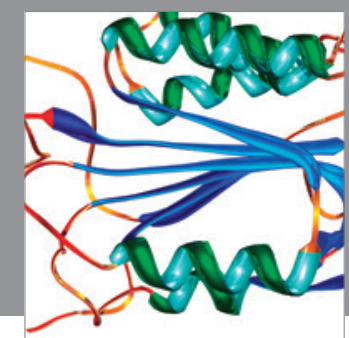

Disease Markers
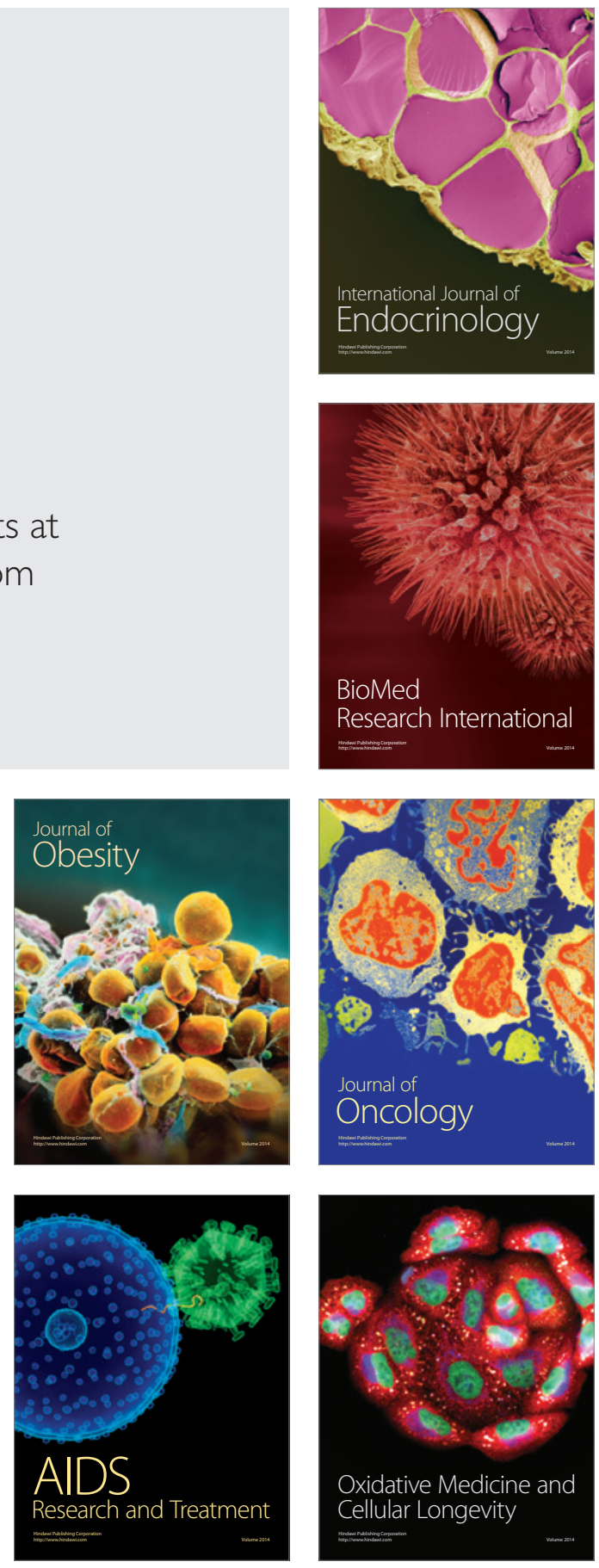